\title{
Where vision begins
}

\author{
Daniele Dell'Orco ${ }^{1} \cdot$ Karl-Wilhelm Koch $^{2} \cdot$ Giorgio Rispoli $^{3}$
}

Published online: 10 July 2021

๑ The Author(s), under exclusive licence to Springer-Verlag GmbH Germany, part of Springer Nature 2021

\section{Introduction}

The vertebrate retina appeared first some 500 million years ago in ancestral marine chordates, evolving since then in a multitude of unique specializations, adapted to the visuoecological lifestyles of each species. The first representation of a vertebrate retina section dates back to 1856 and is due to the German anatomist Heinrich Müller (Fig. 1a), who observed the slices of a fish retina that was fixed and hardened with chromic acid. The morphology of the main retinal cell types and how they are arranged in layers were drawn first in 1887 by the Italian scientist Ferruccio Tartuferi, a pupil of the Nobel prize laureate Camillo Golgi, by staining the retina (freshly dissected from humans, sheep, oxen, dogs, and rabbits) with the Golgi method (Fig. 1b). He was able to construct the first known basic wiring plan of the retina, from the dispositions and arrangements of the individual and clumped cells that had taken up the silver (although the process that select cells and stain them in their entirety is still unknown) [20]. The Tartuferi's diagram identifies correctly the organization of the retinal layers and cells, an amazing accomplishment given the extremely limited knowledge about the retina at his times. Tartuferi agreed with the work of Müller [16] on the vascular shadow perception, with which he identified the "neuroepithelial layer," hosting the rod and cones, the "first site" that is "excited by light" [20]. Obviously, no one at that time could even suspect what is now reported in every textbook, i.e., that vertebrate vision is initiated in the outer segments of the

Giorgio Rispoli

rsg@unife.it

1 Department of Neurosciences, Biomedicine and Movement Sciences, Section of Biological Chemistry, University of Verona, Strada le Grazie 8, I-37124 Verona, Italy

2 Department of Neuroscience, Division of Biochemistry, University of Oldenburg, Carl-von-Ossietzky-Straße 9-11, D-26111 Oldenburg, Germany

3 Department of Neuroscience and Rehabilitation, Section of Physiology, Via Borsari 46, I-44121 Ferrara, Italy rod and cone photoreceptors. Following the absorption of a photon, the visual pigment rhodopsin (Rh, Fig. 2) undergoes several conformational changes to switch to its activated form, metarhodopsin II ( $\left.\mathrm{Rh}^{*}\right)$, which is able to trigger a G-protein-coupled enzymatic cascade. The G-protein transducin (T) targets a specific phosphodiesterase (PDE6) that in turn hydrolyzes the second messenger cGMP leading to the closure of the cyclic nucleotide-gated channels (CNGC). The reduction of the cation influx through these channels, permeable to $\mathrm{Na}^{+}$and $\mathrm{Ca}^{2+}$, causes membrane hyperpolarization and, in turn, a decrease or cessation of neurotransmitter release at the synaptic terminal, which is the message that is relayed to the second-order retinal neurons. This description is however grossly simplified, as shown by the immense number of studies, many of them reviewed in this special issue of the Pflügers Archiv: European Journal of Physiology on "Function and Dysfunction in Vertebrate Photoreceptor Cells." These studies have shed light on the amazing performances of each one of these proteins, on the complex regulation exerted on them by a large number of other ones, and on the several types of ion channels shaping the photoresponse. The concerted action of all these proteins allows the visual system to perform effectively over twelve orders of magnitude of light levels with optimized spatial and temporal resolution. This huge range of light sensitivity is achieved by partitioning the task into the overlapping ranges operated by the slow, highly sensitive rod system (able to detect from the single up to $10^{4}$ absorbed photons) and by the faster, less sensitive cone system (operating over about eight orders of magnitude of light sensitivities). Since the dynamic range of neuronal response in the visual pathway is limited to just three orders of magnitude or less, light adaptation must occur at the photoreceptor level in order to avoid that these neurons exceed their limited dynamic range and saturate. Several mechanisms aimed to reduce the photoreceptor sensitivity as the light intensity increases have been elucidated, but many others are still debated or yet concealed. As expected, several human vision disorders arise from heritable defect in one or more of the proteins 

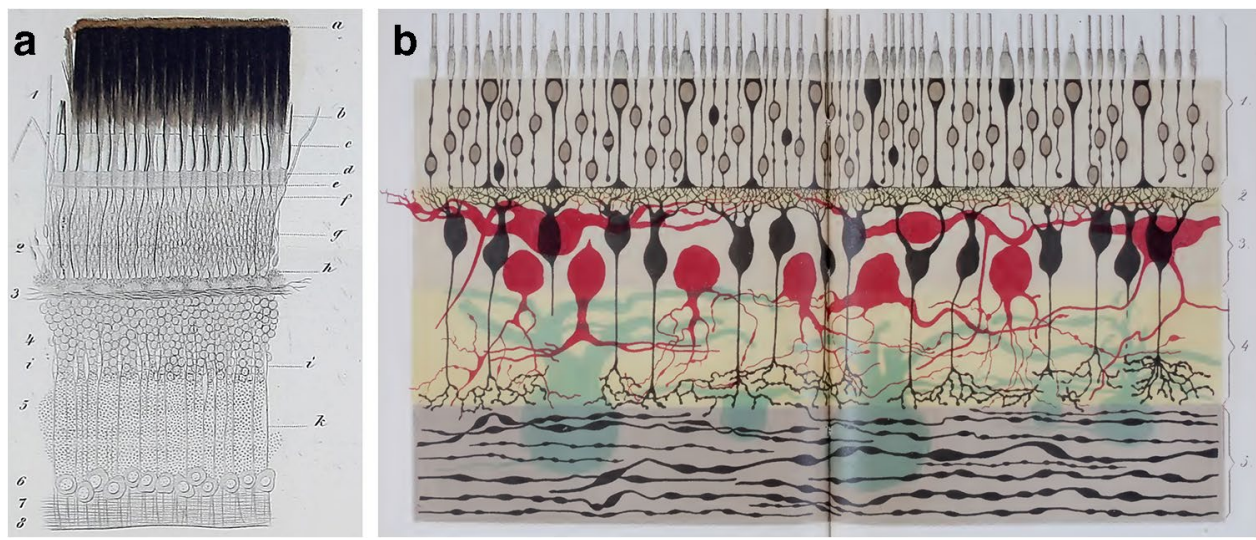

Fig. 1 Historical pictures of phototransduction. a Drawing of a vertical section of a fish (perch) retina as published by Heinrich Müller (1856) (original Fig. 1 of Appendix I; book in German) [16]. The sketch shows the principal layers of a vertebrate retina; the pigment epithelium (a, dark brown) covers most of the rod outer segments (1) and parts of the cone outer segments (b). Clearly visible are the outer nuclear layer (2), the outer plexiform layer (3), the inner nuclear layer (4), a further "granular layer," probably the inner plexiform layer (5), the ganglion cell layer (6, "nerve cell layer"), and the optic nerve fiber layer (7) (b). The first diagram of the retina published by
Ferruccio Tartuferi in 1887 (Table XIX; paper in Italian) [20]. The numbers identify correctly the organization of the retinal layers and cells: 1 , rod and cone photoreceptors, joined to the outer nuclear layer ("neuroepithelial layer" in the original figure legend); 2, outer plexiform layer ("subepithelial layer"); 3, inner nuclear layer, with horizontal and bipolar cells ("cellular portion of the first cerebral layer" endowed with "plumed cells," in black, and with "large superficial cells," in red); 4, inner plexiform layer ("inner reticular layer"); 5, ganglion cell layer, with ganglion cell axons ("layer of the nervous cells," in light blue, "and of the nerve fibers") expressed in photoreceptors, for which therapies remain unfortunately elusive in most cases.

\section{The issue content}

This special issue presents a broad cross-section of meticulous work on the photoreceptor functioning at every level, from the structure-function relationship of single proteins participating in the transduction process to how the entire set of signaling molecules works in concert and generates the photoresponse and the neurotransmitter release. Other key issues, as the molecular origin of the difference between rod and cone photoresponse and the intense molecular trafficking sustaining the rapid phototransduction protein turnover, are also covered.

Following the photon absorption down to synaptic transmission, this special issue virtually begins with two reviews focused on the structure-function relationship of $\mathrm{Rh}$, the light receptor in rods, which consists of a protein milieu
Fig. 2 The membrane ion transport and the phototransduction cascade shared by retinal rods and cones across species. Besides the ubiquitous sustained outward $\mathrm{K}^{+}$current, many other conductances that have been found in rod and cones of different species are omitted. Other regulatory proteins and pathways, as RD3, the $\mathrm{Ca}^{2+}$-regulation of other enzymes besides GCAP and recoverin (REC), and the pigment epithelium processes that resynthesize $\mathrm{Rh}$, are omitted as well for simplicity and/or because are too speculative yet

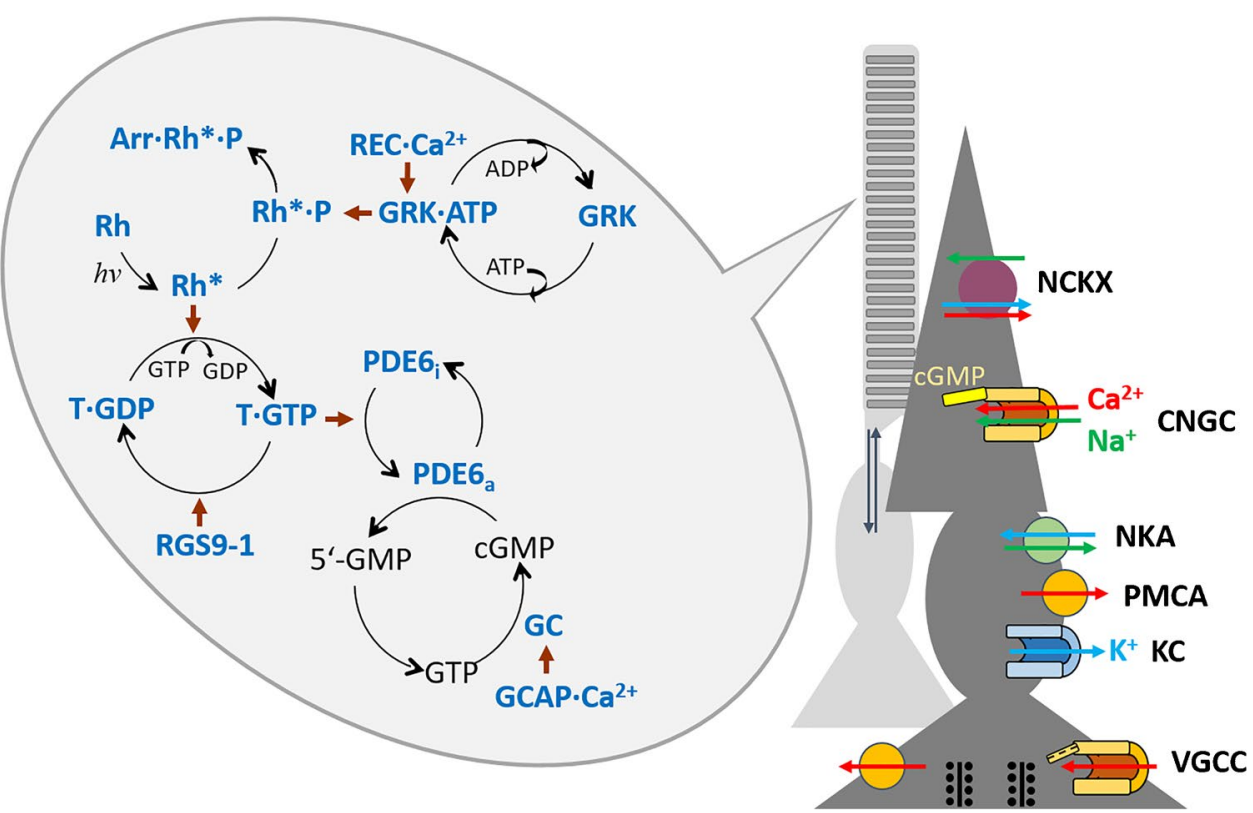


named opsin and the chromophore 11-cis-retinal. Although studies on Rh span well over a century, with many groundbreaking discoveries such as the release of its first crystal structure 20 years ago, questions about the several spontaneous mutations leading to vision impairing diseases and the organization of Rh within the photoreceptor cell membrane are either unresolved or even highly disputed. In particular, the review of Fanelli et al. [8] shows that mutations of rod opsin affect different aspects of its function as folding and trafficking, which have been found to be linked to autosomal dominant or autosomal recessive retinitis pigmentosa and autosomal congenital stationary night blindness. This review also discusses strategies to design small chaperones with therapeutic potential for selected rod opsin mutants.

$\mathrm{Rh}$ deactivation is accomplished by multiple phosphorylation by a specific kinase (GRK), whose activity is modulated by a $\mathrm{Ca}^{2+}$-dependent recoverin (REC), and by binding of arrestin (Arr) to the phosphorylated Rh* (Fig. 2). Rh serves for many years as a textbook example to illustrate a prototypical G-protein-coupled receptor with seven transmembrane $\alpha$-helices spanning a biological membrane. The classical view, depicting $\mathrm{Rh}$ as a monomeric transmembrane receptor that is laterally diffusing in the disk membrane, is however contrasted with the recent evidences presented by Park [18], which support a supramolecular organization of $\mathrm{Rh}$. He compares different experimental and computational approaches that revise the classical view and discusses the physiological necessity of such supramolecular organizations. Possible functional roles for such an organization could also extend to cones, which would explain the very fast cone photoresponse that was recently investigated in zebrafish green cones [1].

In darkness, the photoreceptors are partially depolarized, because of the sustained inward current carried by $\mathrm{Na}^{+}$and $\mathrm{Ca}^{2+}$ (the "dark current") that flows through the CNGC. The concentration in the dark of cGMP in photoreceptor outer segments is maintained by the balance of its continuous synthesis (catalyzed by guanylyl cyclase, GC) and degradation (catalyzed by the PDE6). The $\mathrm{Rh}^{*}$ formed by the light stimulation of Rh triggers the exchange of GTP for GDP on the $\alpha$-subunit of T; T $\alpha$-GTP binds to PDE6 which rapidly increase its basal activity leading to a dramatic drop in cGMP levels (Fig. 2). The review by Cote summarizes recent advances in understanding the molecular mechanism underlying PDE6 activation by the displacement of its $\gamma$-subunits by T $\alpha$-GTP and on the processes regulating the lifetime of activated PDE6 during sustained illumination and following cessation of the light stimulus. A further aspect is the role of the regulator of G-protein signaling 9-1 (RGS9-1; Fig. 2) in controlling the lifetime of T $\alpha$-PDE6 complex [4]. $\mathrm{He}$ also discusses the therapeutic potential of pharmacological compounds targeted on PDE6 to treat inherited retinal diseases resulting from mutations in rod and cone PDE6 genes and/or arising from excessive cGMP accumulation in photoreceptor cytoplasm.

The CNGC closure by the light activation of PDE6 reduces $\mathrm{Ca}^{2+}$ influx and in turn the intracellular $\mathrm{Ca}^{2+}$ concentration, since the rate of $\mathrm{Ca}^{2+}$ extrusion by the outer segment $\mathrm{Na}^{+}: \mathrm{Ca}^{2+}, \mathrm{K}^{+}$exchanger (NCKX; Fig. 2) persists even at low $\mathrm{Ca}^{2+}$ concentrations. $\mathrm{Ca}^{2+}$ has been found to regulate critical steps that are mainly involved in shut-off mechanisms and photoresponse recovery. Specific $\mathrm{Ca}^{2+}$ sensor proteins mediate $\mathrm{Ca}^{2+}$-sensitive regulation of their target proteins, thereby constituting negative feedback loops. These feedback reactions contribute to the control of the phototransduction gain, the cytoplasmic cGMP concentration, and light adaptation. It is a consolidated notion that the $\mathrm{Ca}^{2+}$ fall in light increases the rate of cGMP synthesis through guanylyl cyclase-activating proteins (GCAPs; Fig. 2) [12]: this negative feedback plays a key role in restoring the cGMP concentration once the light stimulation is ceased or to partially recover it during sustained light stimulation; therefore, it is one of the key mechanisms of light adaptation. The paper of Dizhoor and Peshenko [6] reviews the structure of GC and GCAPs in relation to their functional role in phototransduction and in congenital vision diseases. Some forms of congenital retinal degeneration are caused by another regulatory protein named $\mathrm{RD} 3$ that is $\mathrm{Ca}^{2+}$-independent and whose involvement in physiological and disease-associated states is discussed by the authors.

It is expected that any malfunction in proteins involved in the regulation of cytoplasmic $\mathrm{Ca}^{2+}$ and cGMP levels causes an imbalance of their physiological concentrations, thus initiating retinal dysfunction, eventual cell death, and visual impairment. However, the interplay between $\mathrm{Ca}^{2+}$ and cGMP makes it difficult to establish which of the two is most relevant for photoreceptor cell death. The review by Das et al. [5] analyzes the $\mathrm{Ca}^{2+}$-dependent and $\mathrm{Ca}^{2+}$-independent processes that correlate with exceedingly high cytoplasmic cGMP. The authors further discuss the involvement of $\mathrm{Ca}^{2+}$-permeable channels, the general role of energy metabolism, and of calpain-type proteases, which are relevant to the triggering and completion of the photoreceptor cell death. Moreover, the authors briefly review the mutation-independent therapeutic approaches that exploit specific features of cGMP-signaling that might be applied in clinical applications.

The CNGC can be regarded as the "last effector" of the G-protein enzymatic cascade, since their closure leads to cell hyperpolarization, which is the signal that is relayed to the second-order retinal neurons. Tissue-specific CNGC are expressed in several types of sensory cells and appeared very early in the evolution, since they have been found from prokaryotes (including numerous Spirochaeta, although their function in bacterial physiology is still unclear) up to many different cell types across both the animal and the 
plant kingdoms. CNGC have many peculiarities: firstly, they have poor selectivity and do not require membrane depolarization to open, although they share a remarkable amino acid sequence similarity and basic architectural plan with the highly selective, voltage-gated potassium channels. The review of Napolitano et al. [17] focuses on current understanding and controversies on the complex mechanisms coupling gating, the ion permeation process, and its modulation by membrane voltage, on the basis of a wealth of new high-resolution structures of intact CNGC. The authors discuss the structural aspects of this coupling, proposing a model based on the inherent pore flexibility to explain its fundamental properties. Many other channel types shape the voltage light response of photoreceptor cells that is initiated by CNGC closure. These channels set the dark resting potential, extend the photoreceptor dynamic operating range, and trigger the voltage-dependent $\mathrm{Ca}^{2+}$ influx prompting synaptic transmission. Many conductances were found in the photoreceptor inner segment, besides the sustained outward $\mathrm{K}^{+}$current (KC; Fig. 2), which is shared by rod and cones across species. Indeed, a hyperpolarization-activated inward-rectifying current, a sustained voltage-gated $\mathrm{Ca}^{2+}$ current (Cav1.4; VGCC, Fig. 2), a Ca ${ }^{2+}$-activated $\mathrm{Cl}^{-}$current, a $\mathrm{Ca}^{2+}$-activated $\mathrm{K}^{+}$current, and even a voltage-gated $\mathrm{Na}^{+}$current, producing spikes in human rods, have been described, depending upon the species $[10,15]$. The ionic electrochemical gradient of the permeating ions is maintained by a NCKX segregated in the outer segment, a $\mathrm{Na}^{+}: \mathrm{K}^{+}$-ATPase pump (NKA) segregated in the inner segment, and a plasma membrane $\mathrm{Ca}^{2+}$-ATPase pump (PMCA) present in the inner segment and the synaptic terminal (Fig. 2). Of all the above channels, the ones that are chiefly implicated in genetic diseases, are discussed in two reviews $[13,19]$, describing the structure and physiology of $\mathrm{Ca}_{\mathrm{v}} 1.4$, $\mathrm{K}_{\mathrm{v}} 2.1, \mathrm{~K}_{\mathrm{v}} 2.2$, and $\mathrm{K}_{\mathrm{v}} 8.2$ channels and their role in visual processing. Mutations in the genes coding for these channels may lead to pathologies such as cone dystrophy with supernormal rod response and congenital stationary night blindness type 2, leading unfortunately to permanent vision loss. In particular, over 100 mutations in $\mathrm{Ca}_{\mathrm{v}} 1.4$ have been identified, resulting in a spectrum of altered functional channel activity that are associated with X-linked retinal disorders (such as congenital stationary night blindness type 2) and in an altered synapse structure as well. $\mathrm{Ca}_{\mathrm{v}} 1.4$ channels are peculiar to allow the sustained $\mathrm{Ca}^{2+}$ entry at the photoreceptor synapse needed for continuous neurotransmitter release: they activate rapidly at negative membrane potentials of $-40 \mathrm{mV}$, exhibit slow voltage-dependent inactivation, and lack of $\mathrm{Ca}^{2+}$-dependent fast inactivation. Their gating is also controlled by accessory subunits, associated regulatory proteins, and by alternative splicing: the study of these mechanisms, for instance, in knockout mouse models, could be a source of potential specific therapeutic options for patients carrying $\mathrm{Ca}_{\mathrm{v}} 1.4$ mutations, leading also to a deeper understanding of the physiological function of $\mathrm{Ca}_{\mathrm{v}} 1.4$ channels in the retina.

After discussing the enzymatic cascade segregated in the outer segment and the ion channels, distributed in the outer and in the inner segment, this special issue addresses also the third compartment of the photoreceptor, i.e., the synaptic terminal. This very peculiar glutamatergic synapse is endowed with an electron-dense structure known as the ribbon, which holds and conveys vesicles close to the active zone. Besides photoreceptors, the ribbon synapses are found in inner ear hair cells, retinal bipolar cells, and pinealocytes only, i.e., in cell systems where a very fast and sustained neurotransmission is required. The review by Thorenson describes the consolidated notions and what is still debated about this synapse [21], as the role of non-ribbon glutamate release in rods (while it is exclusively released at the cone ribbon sites), the distribution of the vesicle pools, the role of $\mathrm{Ca}_{\mathrm{v}} 1.4$ and $\mathrm{Ca}^{2+}$-activated $\mathrm{Cl}^{-}$channels in the synaptic function, the mechanisms of exocytosis and endocytosis, and the specific properties of ribbon-associated proteins.

The strong photoreceptor compartmentalization, organized in the outer segment, the inner segment, and the presynaptic terminal, is crucial for photoreceptor function. The review by Malhotra et al. describes what is known (and what it is not yet) about the mechanisms that drive the distribution of intrinsic membrane proteins, peripheral membrane proteins, and soluble proteins in these three compartments [14]. The photoreceptor structure, constituted by a large sensory outer segment, a connecting cilium, and a pair of centrioles on which the ciliary axoneme is assembled, contains a path allowing an intense molecular trafficking sustaining rapid protein turnover and its huge energetic demand for phototransduction processes. Any perturbation of this trafficking (indicated in Fig. 2 with a double vertical arrow) leads to devastating blinding diseases known as ciliopathies. Unfortunately, despite decades of study, the details of the fine structure and distribution of molecular components of these structures are still incompletely understood. Recent advances in cellular imaging techniques and animal models of inherited ciliary defects are however yielding important new insights on both of the mechanisms of trafficking and assembly and of the pathophysiological mechanisms of human blindness as discussed in the review by Wensel et al. [22].

Cone photoreceptors provide the foundation of most of the photopic-based human visual experience, although the differences between rod and cones across the species and throughout the evolution do not appear as strong as in mammalian retina. The existence and the physiological role of cone-cone, rod-cone, and rod-rod communication is presented in the review by Cangiano and Asteriti [3] together with a possibly ancestral form of coupling among ciliary 
photoreceptors. The fact that lamprey rods and cones share the same morphology but their photoresponse is as diverse as in the other vertebrates demonstrates that this diversity does not originate from the different surface/volume ratio and/ or from the close proximity of phototransduction enzymes to the CNGC and the exchanger. Instead, it arises from the different kinetic properties of the involved enzymes. The paper of Fain and Sampath [7] addresses this question by identifying the features of the phototransduction cascade that are responsible for the more rapid kinetics and decreased sensitivity of the cone response. Fain and Sampath discuss the roles of inner segment voltage-gated and $\mathrm{Ca}^{2+}$-activated channels, the means by which cones remain responsive even in the brightest illumination, the mechanisms of cone visual pigment regeneration in constant light, and energy consumption of cones in comparison to that of rods.

In recent years, the zebrafish (Danio rerio) has become a favorite model organism for the study of many cellular processes and in particular cone phototransduction [2], because its eye anatomy and physiology exhibit a great homology with other vertebrates and in particular with humans [9]. Moreover, zebrafish embryonic development is remarkably fast [11], and huge resources for its genetic engineering are available. The review by Zang and Neuhauss introduces this model system and its contribution to the understanding of photoreception with a focus on the cone visual transduction cascade [23].

The papers collected in this special issue represent but certainly do not cover the immense amount of work on function and dysfunction in vertebrate photoreceptor cells. Discoveries appear every week heralding new details, often surprising, on the structure/function relationship of the phototransduction proteins and on their regulation and interactions, but as the field progresses, the content of this special issue will constitute a solid framework for guiding future integration.

Acknowledgements We wish to thank all the authors and the journal editors and staff for their fundamental contribution to make this issue possible during such terrible times of COVID-19 pandemic.

\section{References}

1. Aquila M, Benedusi M, Fasoli A, Rispoli G (2015) Characterization of zebrafish green cone photoresponse recorded with pressure-polished patch pipettes, yielding efficient intracellular dialysis. PLoS One 10(10):e0141727. https://doi.org/10.1371/ journal.pone.0141727

2. Aquila M, Dell'Orco D, Fries R, Koch KW, Rispoli G (2019) Incorporating phototransduction proteins in zebrafish green cone with pressure-polished patch pipettes. Biophys Chem 253:106230. https://doi.org/10.1016/j.bpc.2019.106230

3. Cangiano L, Asteriti S (2021) Interphotoreceptor coupling: an evolutionary perspective. Pflügers Arch. https://doi.org/10.1007/ s00424-021-02572-9
4. Cote RH (2021) Photoreceptor phosphodiesterase (PDE6): activation and inactivation mechanisms during visual transduction in rods and cones. Pflügers Arch. https://doi.org/10.1007/ s00424-021-02562-x

5. Das S, Chen Y, Yan J, Christensen G, Belhadj S, Tolone A, Paquet-Durand F (2021) The role of cGMP- and calcium-signalling in photoreceptor cell death: perspectives for therapy development. Pflügers Arch. https://doi.org/10.1007/s00424-021-02556-9

6. Dizhoor AM, Peshenko IV (2021) Regulation of retinal membrane guanylyl cyclase (RetGC) by negative calcium feedback and RD3 protein. Pflügers Arch. https://doi.org/10.1007/ s00424-021-02523-4

7. Fain GL, Sampath AP (2021) Light responses of mammalian cones. Pflügers Arch. https://doi.org/10.1007/s00424-021-02551-0

8. Fanelli F, Felline A, Marigo V (2021) Structural aspects of rod opsin and their implication in genetic diseases. Pflügers Arch. https://doi.org/10.1007/s00424-021-02546-x

9. Gestri G, Link BA, Neuhauss SC (2012) The visual system of zebrafish and its use to model human ocular diseases. Dev Neurobiol 72(3):302-327. https://doi.org/10.1002/dneu.20919

10. Kawai F, Horiguchi M, Suzuki H, Miyachi E (2001) Na+ action potentials in human photoreceptors. Neuron 30(2):451-458. https://doi.org/10.1016/S0896-6273(01)00299-9

11. Kimmel CB, Ballar WW, Kimme SR, Ullmann B, Schilling TF (1995) Stages of embryonic development of the zebrafish. Dev Dyn 203:253-310. https://doi.org/10.1002/aja.1002030302

12. Koch KW, Dell'Orco D (2013) A calcium relay mechanism in vertebrate phototransduction. ACS Chem Neurosci 4(6):909-917. https://doi.org/10.1021/cn400027z

13. Koschak A, Quintero MF, Heigl T, Ruzza M, Seitter H, Zanetti L (2021) Cav1.4 dysfunction and congenital night blindness type 2 . Pflügers Arch. https://doi.org/10.1007/s00424-021-02570-x

14. Malhotra H, Barnes CL, Calvert PD (2021) Functional compartmentalization of photoreceptor neurons. Pflügers Arch. In press

15. Moriondo A, Rispoli G (2010) The contribution of cationic conductances to the potential of rod photoreceptors. Eur Biophys J 39(6):889-902. https://doi.org/10.1007/s00249-009-0419-z

16. Müller H (1856) Anatomisch-physiologische Untersuchungen über die Retina des Menschen und der Wirbelthiere. Leipzig, Wilhelm Engelmann. https://wellcomecollection.org/works/qzr2vw47

17. Napolitano LMR, Torre V, Marchesi A (2021) CNG channel structure, function, and gating: a tale of conformational flexibility. Pflügers Arch. In press

18. Park PS (2021) Supramolecular organization of rhodopsin in rod photoreceptor cell membranes. Pflügers Arch. https://doi.org/10. 1007/s00424-021-02522-5

19. Rashwan R, Hunt DM, Carvalho LS (2021) The role of voltagegated ion channels in visual function and disease in mammalian photoreceptors. Pflügers Arch. In press

20. Tartuferi F (1887) Sull'anatomia della retina. Internationale Monatsschrift für Anatomie und Physiologie. 4(10):421-441. https://www.biodiversitylibrary.org/item/110302\#page/9/mode/ lup

21. Thoreson WB (2021) Transmission at rod and cone ribbon synapses in the retina. Pflügers Arch. https://doi.org/10.1007/ s00424-021-02548-9

22. Wensel TG, Potter VL, Moye A, Zhang Z, Robichaux MA (2021) Structure and dynamics of photoreceptor sensory cilia. Pflügers Arch. https://doi.org/10.1007/s00424-021-02564-9

23. Zang J, Neuhauss SCF (2021) Biochemistry and physiology of zebrafish photoreceptors. Pflügers Arch. https://doi.org/10.1007/ s00424-021-02528-z

Publisher's note Springer Nature remains neutral with regard to jurisdictional claims in published maps and institutional affiliations. 